\title{
A Family of Quadratic Snakes for Road Extraction
}

\author{
Ramesh Marikhu ${ }^{1}$ Matthew N. Dailey ${ }^{2}$ Stanislav Makhanov ${ }^{3}$ Kiyoshi Honda ${ }^{4}$ \\ 1 Information and Communication Technologies, Asian Institute of Technology \\ 2 Computer Science and Information Management, Asian Institute of Technology \\ 3 Sirindhorn International Institute of Technology, Thammasat University \\ ${ }^{4}$ Remote Sensing and GIS, Asian Institute of Technology
}

\begin{abstract}
The geographic information system industry would benefit from flexible automated systems capable of extracting linear structures from satellite imagery. Quadratic snakes allow global interactions between points along a contour, and are well suited to segmentation of linear structures such as roads. However, a single quadratic snake is unable to extract disconnected road networks and enclosed regions. We propose to use a family of cooperating snakes, which are able to split, merge, and disappear as necessary. We also propose a preprocessing method based on oriented filtering, thresholding, Canny edge detection, and Gradient Vector Flow (GVF) energy. We evaluate the performance of the method in terms of precision and recall in comparison to ground truth data. The family of cooperating snakes consistently outperforms a single snake in a variety of road extraction tasks, and our method for obtaining the GVF is more suitable for road extraction tasks than standard methods.
\end{abstract}

\section{Introduction}

The geographic information system industry would benefit from flexible automated systems capable of extracting linear structures and regions of interest from satellite imagery. In particular, automated road extraction would boost the productivity of technicians enormously. This is because road networks are among the most important landmarks for mapping, and manual marking and extraction of road networks is an extremely slow and laborious process. Despite years of research and significant progress in the computer vision and image processing communities (see, for example, [1,2] and Fortier et al.'s survey [3]), the methods available thus far have still not attained the speed and accuracy necessary for practical application in GIS tools.

Among the most promising techniques for extraction of complex objects like roads are active contours or snakes, originally introduced by Kass et al. [4]. Since the seminal work of Kass and colleagues, techniques based on active contours have been applied to many object extraction tasks [5] including road extraction [6].

Rochery et al. have recently proposed higher-order active contours, in particular quadratic snakes, which hold a great deal of promise for extraction of linear 
structures like roads [7]. The idea is to use a quadratic formulation of the contour's geometric energy to encourage anti-parallel tangents on opposite sides of a road and parallel tangents along the same side of a road. These priors increase the final contour's robustness to partial occlusions and decrease the likelihood of false detections in regions not shaped like roads.

In this paper, we propose two heuristic modifications to Rochery et al.'s quadratic snakes, to address limitations of a single quadratic snake and to accelerate convergence to a solution. First, we introduce the use of a family of quadratic snakes that are able to split, merge, and disappear as necessary. Second, we introduce an improved formulation of the image energy combining Rochery et al.'s oriented filtering technique [7] with thresholding, Canny edge detection, and Xu and Prince's Gradient Vector Flow (GVF) [8]. The modified GVF field created using the proposed method is very effective at encouraging the quadratic snake to snap to the boundaries of linear structures. We demonstrate the effectiveness of the family of snakes and the modified GVF field in a series of experiments with real satellite images, and we provide precision and recall measurements in comparison with ground truth data. The results are an encouraging step towards the ultimate goal of robust, fully automated road extraction from satellite imagery.

As a last contribution, we have developed a complete GUI environment for satellite image manipulation and quadratic snake evolution, based on the Matlab platform. The system is freely available as open source software [9].

\section{Experimental Methods}

\subsection{Quadratic snake model}

Here we provide a brief overview of the quadratic snake proposed by Rochery et al. [7]. An active contour or snake is parametrically defined as

$$
\gamma(p)=[x(p) y(p)]^{T},
$$

where $p$ is the curvilinear abscissa of the contour and the vector $[x(p) y(p)]^{T}$ defines the Cartesian coordinates of the point $\gamma(p)$. We assume the image domain $\Omega$ to be a bounded subset of $\mathbb{R}^{2}$.

The energy functional for Rochery et al.'s quadratic snake is given by

$$
E_{s}(\gamma)=E_{g}(\gamma)+\lambda E_{i}(\gamma)
$$

where $E_{g}(\gamma)$ is the geometric energy and $E_{i}(\gamma)$ is the image energy of the contour $\gamma . \lambda$ is a free parameter determining the relative importance of the two terms.

The geometric energy functional is defined as

$$
E_{g}(\gamma)=L(\gamma)+\alpha A(\gamma)-\frac{\beta}{2} \iint \boldsymbol{t}_{\boldsymbol{\gamma}}(p) \cdot \boldsymbol{t}_{\boldsymbol{\gamma}}\left(p^{\prime}\right) \Psi\left(\left\|\gamma(p)-\gamma\left(p^{\prime}\right)\right\|\right) d p d p^{\prime}
$$

where $L(\gamma)$ is the length of $\gamma$ in the Euclidean metric over $\Omega, A(\gamma)$ is the area enclosed by $\boldsymbol{\gamma}, \boldsymbol{t}_{\boldsymbol{\gamma}}(p)$ is the unit-length tangent to $\boldsymbol{\gamma}$ at point $p$, and $\Psi(z)$, given the 
distance $z$ between two points on the contour, is used to weight the interaction between those two points (see below). $\alpha$ and $\beta$ are constants weighting the relative importance of each term. Clearly, for positive $\beta, E_{g}(\gamma)$ is minimized by contours with short length and parallel tangents. If $\alpha$ is positive, contours with small enclosed area are favored; if it is negative, contours with large enclosed area are favored.

The interation function $\Psi(z)$ is a smooth function expressing the radius of the region in which parallel tangents should be encouraged and anti-parallel tangents should be discouraged. $\Psi(z)$ incorporates two constants: $d$, the expected road width, and $\epsilon$, the expected variability in road width. During snake evolution, weighting by $\Psi(z)$ in Equation 3 discourages two points with anti-parallel tangents (the opposite sides of a putative road) from coming closer than distance $d$ from each other.

The image energy functional $E_{i}(\gamma)$ is defined as

$$
\begin{aligned}
E_{i}(\boldsymbol{\gamma})= & \int \boldsymbol{n}_{\boldsymbol{\gamma}}(p) \cdot \nabla I(\boldsymbol{\gamma}(p)) d p \\
& -\iint \boldsymbol{t}_{\boldsymbol{\gamma}}(p) \cdot \boldsymbol{t}_{\boldsymbol{\gamma}}\left(p^{\prime}\right) \nabla I(\gamma(p)) \cdot \nabla I\left(\boldsymbol{\gamma}\left(p^{\prime}\right)\right) \Psi\left(\left\|\gamma(p)-\gamma\left(p^{\prime}\right)\right\|\right) d p d p^{\prime},
\end{aligned}
$$

where $I: \Omega \rightarrow[0,255]$ is the image and $\nabla I(\gamma(p))$ denotes the $2 \mathrm{D}$ gradient of $I$ evaluated at $\gamma(p)$. The first linear term favors anti-parallel normal and gradient vectors, encouraging counterclockwise snakes to shrink around or clockwise snakes to expand to enclose dark regions surrounded by light roads. ${ }^{5}$ The quadratic term favors nearby point pairs with two different configurations, one with parallel tangents and parallel gradients and the other with anti-parallel tangents and anti-parallel gradients.

After solving the Euler-Lagrange equations for minimizing the energy functional $E_{s}(\gamma)$ (Equation 2), Rochery et al. obtain the update equation

$$
\begin{aligned}
& \boldsymbol{n}_{\boldsymbol{\gamma}}(p) \cdot \frac{\partial E_{s}}{\partial \boldsymbol{\gamma}}(p)=-\kappa_{\boldsymbol{\gamma}}(p)-\alpha-\lambda\|\nabla I(\gamma(p))\|^{2}+G(\gamma(p)) \\
& \quad+\beta \int \boldsymbol{r}\left(\boldsymbol{\gamma}(p), \gamma\left(p^{\prime}\right)\right) \cdot \boldsymbol{n}_{\boldsymbol{\gamma}}\left(p^{\prime}\right) \Psi^{\prime}\left(\left\|\gamma(p)-\gamma\left(p^{\prime}\right)\right\|\right) d p^{\prime} \\
& +2 \lambda \int \boldsymbol{r}\left(\boldsymbol{\gamma}(p), \gamma\left(p^{\prime}\right)\right) \cdot \boldsymbol{n}_{\boldsymbol{\gamma}}\left(p^{\prime}\right)\left(\nabla I(\gamma(p)) \cdot \nabla I\left(\gamma\left(p^{\prime}\right)\right)\right) \Psi^{\prime}\left(\left\|\gamma(p)-\gamma\left(p^{\prime}\right)\right\|\right) d p^{\prime} \\
& \quad+2 \lambda \int \nabla I\left(\gamma\left(p^{\prime}\right)\right) \cdot\left(\nabla \nabla I(\gamma(p)) \times \boldsymbol{n}_{\boldsymbol{\gamma}}\left(p^{\prime}\right)\right) \Psi\left(\left\|\gamma(p)-\gamma\left(p^{\prime}\right)\right\|\right) d p^{\prime}, \quad(5)
\end{aligned}
$$

where $\kappa_{\boldsymbol{\gamma}}(p)$ is the curvature of $\boldsymbol{\gamma}$ at $\boldsymbol{\gamma}(p)$ and $G(\boldsymbol{\gamma}(p))$ is the "specific energy," evaluated at point $\gamma(p)$ (Section 2.2). $\boldsymbol{r}\left(\gamma(p), \gamma\left(p^{\prime}\right)\right)=\frac{\gamma(p)-\gamma\left(p^{\prime}\right)}{\left\|\gamma(p)-\gamma\left(p^{\prime}\right)\right\|}$ is the unit

\footnotetext{
${ }^{5}$ For dark roads in light background, we negate all the terms involving image, including $G(\gamma(p))$ in Equation 5. In the rest of the paper, we assume light roads on a dark background.
} 
vector pointing from $\gamma(p)$ towards $\gamma\left(p^{\prime}\right) . \nabla \nabla I(\gamma(p))$ is the Hessian of $I$ evaluated at $\gamma(p)$.

$\alpha, \beta$, and $\lambda$ are free parameters that need to be determined experimentally. $d$ and $\epsilon$ are specified a priori according to the desired road width. Following Rochery et al., we normally initialize our quadratic snakes with a rounded rectangle covering the entire image.

\subsection{Oriented filtering}

We use Rochery's oriented filtering method [10] to enhance linear edges in our satellite imagery. The input image is first convolved with oriented derivativeof-Gaussian filters at various orientations. Then the minimum (most negative) filter response over the orientations is run through a ramp function equal to 1 for low filter values and -1 for high filter values. The thresholds are user-specified. An example is shown in Fig. 1(b).

\section{$2.3 \quad$ GVF energy}

Rather than using the oriented filtering specific image energy $G(\boldsymbol{x})$ from Section 2.2 for snake evolution directly, we propose to combine the oriented filtering approach with Xu and Prince's Gradient Vector Flow (GVF) method [8]. The GVF is a vector field $V^{\mathrm{GVF}}(\boldsymbol{x})=[u(\boldsymbol{x}) v(\boldsymbol{x})]^{T}$ minimizing the energy functional

$$
\begin{array}{r}
E\left(V^{\mathrm{GVF}}\right)=\int_{\Omega} \mu\left(u_{x}^{2}(\boldsymbol{x})+u_{y}^{2}(\boldsymbol{x})+v_{x}^{2}(\boldsymbol{x})+v_{y}^{2}(\boldsymbol{x})\right) \\
+\|\nabla \tilde{I}(\boldsymbol{x})\|^{2}\|V(\boldsymbol{x})-\nabla \tilde{I}(\boldsymbol{x})\|^{2} d \boldsymbol{x},
\end{array}
$$

where $u_{x}=\frac{\partial u}{\partial x}, u_{y}=\frac{\partial u}{\partial y}, v_{x}=\frac{\partial v}{\partial x}, v_{y}=\frac{\partial v}{\partial y}$, and $\tilde{I}$ is a preprocessed version of image $I$, typically an edge image of some kind. The first term inside the integral encourages a smooth vector field whereas the second term encourages fidelity to $\nabla \tilde{I} . \mu$ is a free parameter controlling the relative importance of the two terms.

$\mathrm{Xu}$ and Prince [8] experimented with several different methods for obtaining $\nabla \tilde{I}$. We propose to perform Canny edge detection on $G$ (the result of oriented filtering and thresholding, introduced in Section 2.2) to obtain a binary image $\tilde{I}$ for GVF, then to use the resulting GVF $V^{\mathrm{GVF}}$ as an additional image energy for quadratic snake evolution. The binary Canny image is ideal because it only includes information about road-like edges that have survived sharpening by oriented filters. The GVF field is ideal because during quadratic snake evolution, it points toward road-like edges, pushing the snake in the right direction from a long distance away. This speeds evolution and makes it easier to find suitable parameters to obtain fast convergence. Fig. 1 compares our method to alternative GVF formulations based on oriented filtering or Canny edge detection alone. 


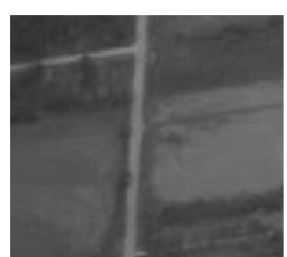

(a)

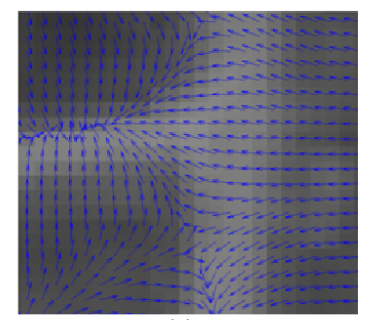

(e)

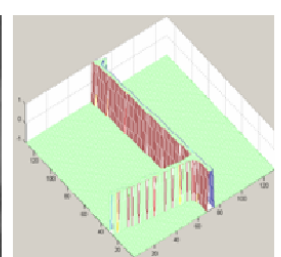

(b)
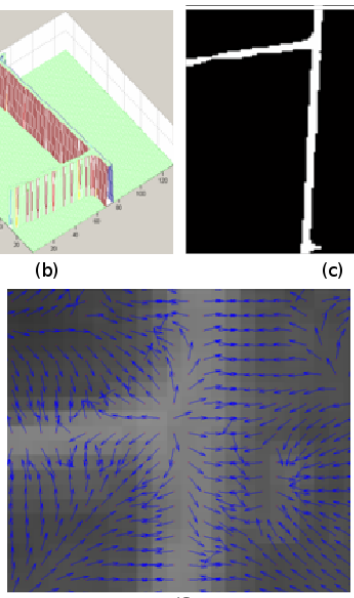

(f)
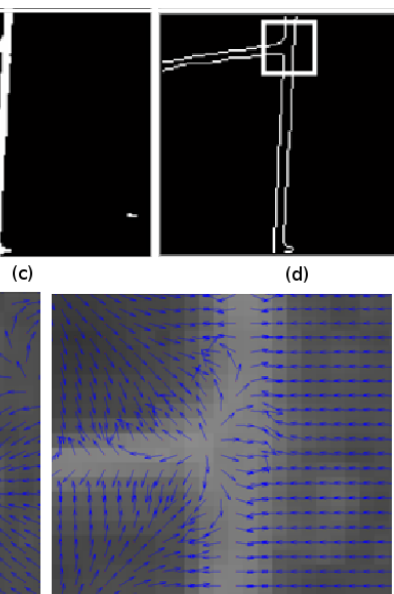

(g)

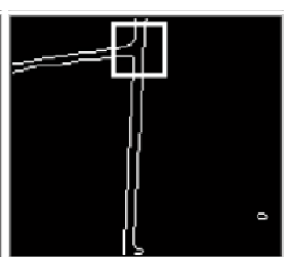

(d)

Fig. 1. Comparison of GVF methods. (a) Input image. (b) $G(\boldsymbol{x})$ obtained from oriented filtering on $I(\boldsymbol{x})$. (c) Image obtained from $G(\boldsymbol{x})$ using threshold 0. (d) Canny edge detection on (c), used as $\tilde{I}$ for GVF. (e-f) Zoomed views of GVFs in region delineated in (d). (e) Result of using the magnitude of the gradient $\nabla\left(G_{\sigma} * I\right)$ to obtain $\tilde{I}$. (f) Result of using Canny edge detection alone to obtain $\tilde{I}$. (g) GVF energy obtained using our proposed edge image. This field pushes most consistently toward the true road boundaries.

\subsection{Family of quadratic snakes}

A single quadratic snake is unable to extract enclosed regions and multiple disconnected networks in an image. We address this limitation by introducing a family of cooperating snakes that are able to split, merge, and disappear as necessary.

In our formulation, due to the curvature term $\kappa_{\gamma}(p)$ and the area constant $\alpha$ in Equation 5, specifying the points on $\gamma$ in a counterclockwise direction creates a shrinking snake and specifying the points on $\gamma$ in a clockwise direction creates a growing snake.

An enclosed region (loop or a grid cell) can be extracted effectively by initializing two snakes, one shrinking snake covering the whole road network and another growing snake inside the enclosed region.

On the one hand, our method is heuristic and dependent on somewhat intelligent user initialization, but it is much simpler than level set methods for the same problem [7], and, assuming a constant number of splits and merges per iteration, it does not increase the asymptotic complexity of the quadradic snake's evolution.

Splitting a snake We split a snake into two snakes whenever two of its arms are squeezed too close together, i.e. when the distance between two snake points is less than $d^{\text {split }}$ and those two points are at least $k$ snake points from each other 
in both directions of traversal around the contour. $d^{\text {split }}$ should be less than $2 \eta$, where $\eta$ is the maximum step size.

Merging two snakes Two snakes are merged when they have high curvature points within a distance $d^{\text {merge }}$ of each other, the two snakes' order of traversal (clockwise or counterclockwise) is the same, and the tangents at the two high curvature points are nearly antiparallel. High curvature points are those with $\kappa_{\gamma}(p)>0.6 \kappa_{\gamma}^{\max }$ where $\kappa_{\gamma}^{\max }$ is the maximum curvature for any point on $\gamma$. High curvature points are taken to ensure merging only occurs if two snakes have the semi-circular tip of their arms facing each other. Filtering out the low curvature points necessitates computing angle between the tangents at two points only for the high curvature points.

When these conditions are fulfilled, the two snakes are merged by deleting the high curvature points and joining the snakes into a single snake while preserving the direction of traversal for the combined snake.

Deleting a snake A snake $\gamma$ is deleted if it has low compactness $\left(\frac{4 \pi A(\gamma)}{L(\gamma)^{2}}\right)$ and a perimeter less than $L^{\text {delete }}$.

\subsection{Experimental design}

We analyze extraction results on different types of road networks using the single quadratic snake proposed by Rochery et al. [7] and the proposed family of cooperating snakes. The default convergence criterion is when the minimum $E_{s}(\gamma)$ has not improved for some number of iterations.

Experiments have been performed to analyze the extraction of tree-structured road networks and those with loops, grids and disconnected networks.

We then analyze the effectiveness of GVF energy obtained from the proposed edge image in Experiment 4. For all the experiments, we digitize the images manually to obtain the ground truth data necessary to compute precision and recall.

\section{$3 \quad$ Results}

We have obtained several parameters emperically. For splitting a snake, $d^{\text {split }}$ should be less than d. $k$ to be chosen depending on how far the two splitting points should be to ensure that the snakes formed after splitting have at least $k$ points.

In order to ensure that merging of snakes takes place only among the arms with the semi-circular tips facing each other, the tangents at the high curvature points are checked for antiparallel threshold of $130 \pi / 180$.

The compactness should be greater than 0.2 to ensure that linear structured contours are not deleted. 

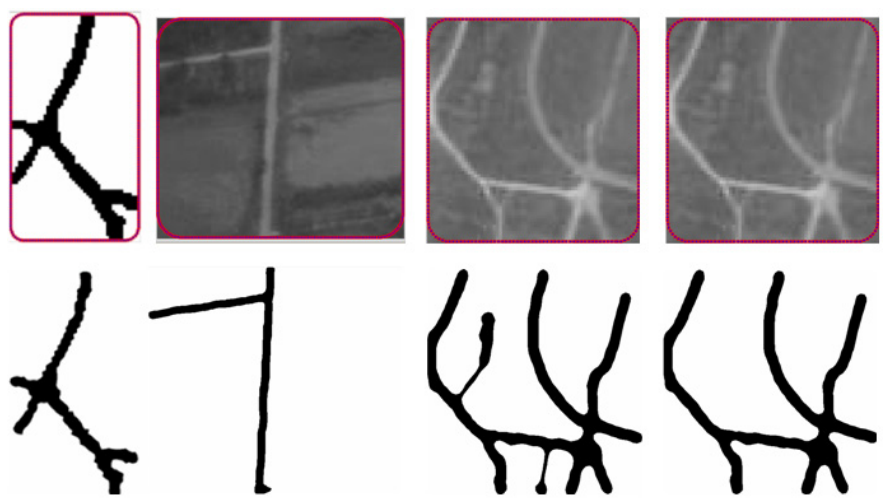

\begin{tabular}{|c|c|c|c|c|c|c|c|c|}
\hline Column & Snakes & Iter & Precision & Recall & $\alpha$ & $\beta$ & $\lambda$ & $\mathbf{d}$ \\
\hline 1 & single & 480 & 0.937 & 0.8605 & 0.75 & 0.35 & 4 & 3 \\
\hline 2 & single & 308 & 0.9483 & 0.8503 & 0.15 & 0.2 & 3.5 & 4 \\
\hline 3 & single & 473 & 0.7091 & 0.9074 & 0.1 & 0.2 & 4 & 3 \\
\hline 4 & family & 450 & 0.8011 & 0.9115 & 0.1 & 0.2 & 4 & 3 \\
\hline
\end{tabular}

Fig. 2. Evolution of quadratic snake on roads with tree structure.Each column displays an image with initial contour in red and the extracted road network below it.

\subsection{Experiment 1: Simple (tree-structured) road networks}

A single quadratic snake is well suited for tree-structured road networks as the snake will not need to change its topology during evolution (Figure 2). A family of snakes enable faster and better road extraction as non-road regions are eliminated using splitting and deletion of snakes.

\subsection{Experiment 2: Road networks with single loop and multiple disconnected networks}

The family of quadratic snakes are able to extract disconnected networks with high accuracy (Figure 3 ) but are not able to extract enclosed regions automatically as the snakes are not able to develop holes inside it in the form of growing snakes.

\subsection{Experiment 3: Complex road networks}

A road network is considered complex if it has multiple disconnected networks and enclosed regions and large number of branches. With the appropriate user initialization (Figure 4), the snakes are able to extract the road networks with high accuracy and in less time. 


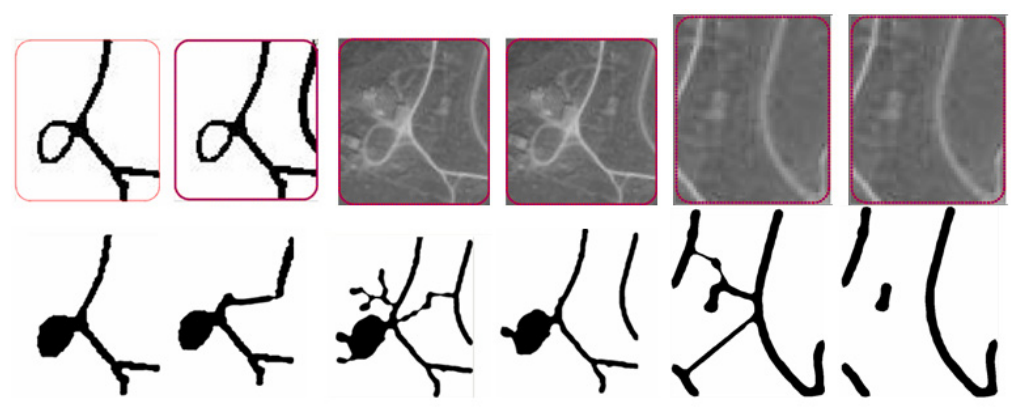

\begin{tabular}{|c|c|l|c|c|c|c|c|c|}
\hline Column & Snakes & Iter & Precision & Recall & $\alpha$ & $\beta$ & $\lambda$ & $\mathbf{d}$ \\
\hline 1 & single & 1080 & 0.6364 & 0.9833 & 0.75 & 0.35 & 4 & 3 \\
\hline 2 & single & 1300 & 0.5638 & 0.7322 & 0.75 & 0.35 & 4 & 3 \\
\hline 3 & single & 182 & 0.5473 & 0.9114 & 0.1 & 0.2 & 4 & 2 \\
\hline 4 & family & 182 & 0.6344 & 0.9833 & 0.1 & 0.2 & 4 & 2 \\
\hline 5 & single & 219 & 0.6823 & 0.8673 & 0.1 & 0.2 & 3 & 2.5 \\
\hline 6 & family & 163 & 0.8582 & 0.8731 & 0.1 & 0.2 & 3 & 2.5 \\
\hline
\end{tabular}

Fig. 3. Evolution of quadratic snake on roads with loops and disconnected networks. Each column displays an image with initial contour in red and the extracted road network below it.

\subsection{Experiment 4: GVF energy to enable faster evolution}

The Gradient Vector Flow Field [8] boosts the evolution process as we can see from the number of iterations required for each evolution in Experiment 4 with and without the use of GVF energy. From the evolution in the fifth column, we see that the snake was able to extract the network with greater detail. Also, from the evolution in the last column, we see that it is necessary for the quadratic image energy to enable robust extraction and thus the GVF weight and $\lambda$ need to be balanced appropriately.

\section{Discussion and Conclusion}

In Experiment 1, we found that the our modified quadratic snake is able to move into concavities to extract entire tree-structured road networks with very high accuracy. Experiment 2 showed that the family of quadratic snakes is effective at handling changes in topology during evolution, enabling better extraction of road networks. Currently, loops cannot be extracted automatically.

We demonstrated the difficulty in extracting complex road networks with multiple loops and grids in Experiment 3. However, user initialization of a family of contours enable extraction of multiple closed regions and help the snake to avoid road-like regions. The level set framework could be used to handle change in topology enabling effective extraction of enclosed regions. Rochery et al. [10] evolved the contour using the level set methods introduced by Osher and Sethian. 


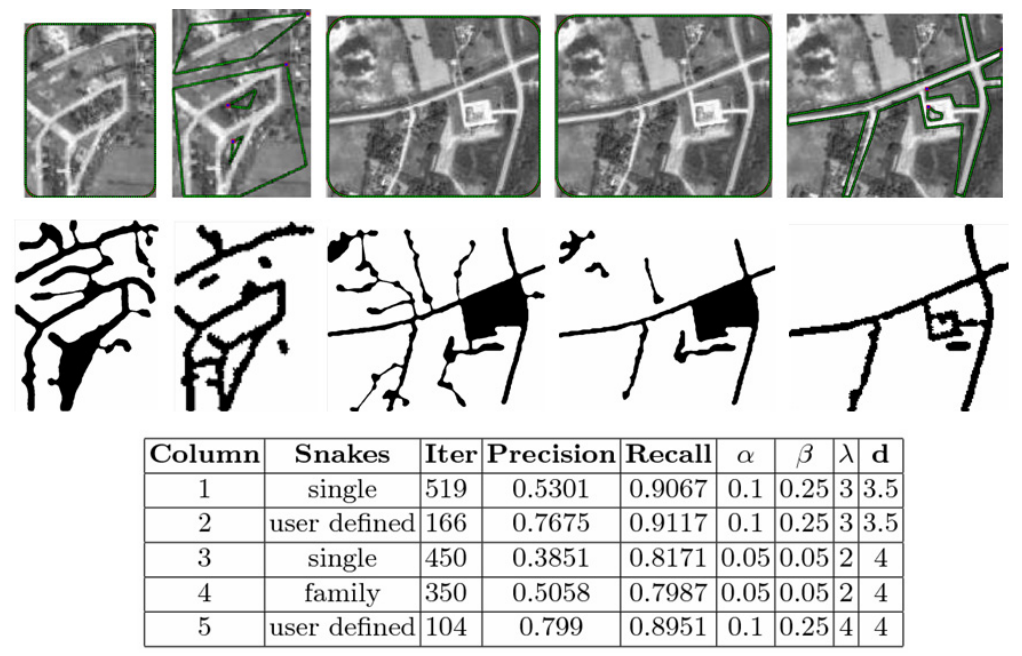

Fig. 4. Evolution of quadratic snake on roads with enclosed regions. Each column displays an image with initial contour in green and the extracted road network below it.

However, our method is faster, conceptually simpler, and a direct extension of Kass et al.'s computational approach.

In Experiment 4, we found that faster and robust extraction is achieved using oriented filtering and GVF energy along with image energy of the quadratic snakes. Our proposed edge image obtained from oriented filtering is effective for computing GVF energy to enhance the process of extraction. We also found that our method for obtaining the GVF outperforms standard methods.

Finally, we have developed a complete GUI environment for satellite image manipulation and quadratic snake evolution, based on the Matlab platform. The system is freely available as open source software [9].

Future work will focus on possibilities to automate the extraction of enclosed regions. Digital elevation models could be integrated with image energy for increased accuracy.

\section{Acknowledgments}

This research was supported by Thailand Research Fund grant MRG4780209 to MND. RM was supported by a graduate fellowship from the Nepal High Level Commission for Information Technology.

\section{References}

1. Fischler, M., Tenenbaum, J., Wolf, H.: Detection of roads and linear structures in low-resolution aerial imagery using a multisource knowledge integration technique. Computer Graphics and Image Processing 15 (1981) 201-223 


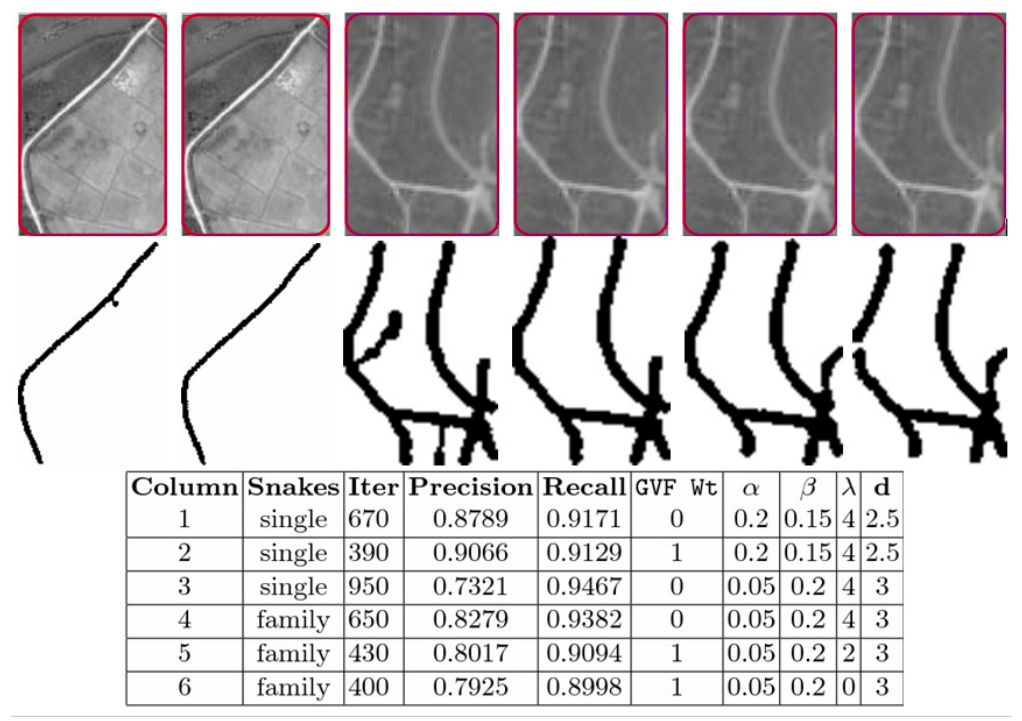

Fig. 5. Evolution of quadratic snake on roads with enclosed regions. Each column displays an image with initial contour in green and the extracted road network below it.

2. Geman, D., Jedynak, B.: An active testing model for tracking roads in satellite images. IEEE Transactions on Pattern Analysis and Machine Intelligence 18(1) (1996) 1-14

3. Fortier, A., Ziou, D., Armenakis, C., Wang, S.: Survey of work on road extraction in aerial and satellite images. Technical Report 241, Université de Sherbrooke, Quebec, Canada (1999)

4. Kass, M., Witkin, A., Terzopoulos, D.: Snakes: Active contour models. International Journal of Computer Vision 1(4) (1987) 321-331

5. Cohen, L.D., Cohen, I.: Finite-element methods for active contour models and balloons for 2-D and 3-D images. IEEE Transactions on Pattern Analysis and Machine Intelligence 15(11) (1993) 131-147

6. Laptev, I., Mayer, H., Lindeberg, T., Eckstein, W., Steger, C., Baumgartner, A.: Automatic extraction of roads from aerial images based on scale space and snakes. Machine Vision and Applications 12(1) (2000) 23-31

7. Rochery, M., Jermyn, I.H., Zerubia, J.: Higher order active contours. International Journal of Computer Vision 69(1) (2006) 27-42

8. $\mathrm{Xu}, \mathrm{C}$., Prince, J.L.: Gradient Vector Flow: A new external force for snakes. In: IEEE Conference on Computer Vision and Pattern Recognition (CVPR). (1997) $66-71$

9. Marikhu, R.: A GUI environment for road extraction with quadratic snakes (2007) Matlab software, available at http://www.cs.ait.ac.th/ mdailey/snakes.

10. Rochery, M.: Contours actifs d'order supérieur et leur application à la détection de linéiques dans des images de télédétection. PhD thesis, Université de Nice, Sophia Antipolis — UFR Sciences (2005) 